\title{
Unusual Olecranon Swelling - Epidermoid Cyst of Elbow
}

\section{Abstract}

Epidermoid cysts are relatively common entities, but we present a large cyst which occurred almost directly over the olecranon, a previously unreported location. The 31 year old male patient had a history of previous epidermoid cysts in the head/ neck and after en block excision there has been no recurrence at 4 years.

Keywords: Olecranon process; Elbow joint; Epidermoid cyst

Received: January 06, 2016; Accepted: January 18, 2016; Published: January 26, 2016

\section{Introduction}

Epidermoid cysts, also called epidermal cysts, epidermal inclusion cysts, or infundibular cysts, are a common benign intradermal lesion, which can occur virtually anywhere in the body [1-3]. They represent $85-90 \%$ of all excised cysts [4]. Like all true cysts, they are lined by epithelium, specifically stratified squamous epithelium [5].They are generally painless, firm, subcutaneous nodules that are filled with keratin debris $[2,5]$. No racial predilection to developing epidermoid cysts has been identified, but they are about twice as common in males as in females [1]. They are most commonly seen in the third and fourth decades of life, but can be seen at any age [1]. Although they can occur anywhere in the body, epidermoid cysts are most frequently seen on (in order of descending frequency) the face, trunk, neck, extremities and scalp [1]. The orthopedist most frequently encounters them on the volar palm and digits, as they are common soft tissue lesions of the hand [2].

There are several mechanisms through which these cysts can result, but all have the common endpoint of having epithelial elements being implanted into deeper tissues. This can be a result of epidermal rests sequestered during embryonic life, occlusion of the pilosebaceous unit, or traumatic or surgical implantation $[1,2,6]$. Theoretically, any surgical procedure could result in the formation of an epidermoid cyst and their formation has been associated with dermal and myocutaneous grafts as well as needle biopsies [1].

Epidermoid cysts do have the potential to form inside bone. This is frequently seen in the terminal phalanx where a history of trauma such as having had the finger slammed in a door is common [1]. When investigated with plain radiography, they present as radiolucent lytic bone lesions with clearly defined boundaries that expand the cortex of the bone $[5,7]$.

\section{Srinath Kamineni and Danielle Reilly}

\author{
University of Kentucky, Department of \\ Orthopaedic Surgery, Elbow Shoulder \\ Research Centre, USA
}

Corresponding author: Dr. Srinath Kamineni

srinathkamineni@gmail.com

University of Kentucky, Department of Orthopaedic Surgery, Kentucky Clinic, 740 South Limestone Street, K412, Lexington KY 40536, USA.

Tel: (859) 218-3057

Fax: (859) 323-2412

Citation: Kamineni S, Reilly D. Unusual Olecranon Swelling - Epidermoid Cyst of Elbow. Bone Rep Recommendations. 2016, 2:1.

Although these are benign lesions, they do have a potential for malignant transformation. There is a handful of case reports in the current literature documenting squamous cell carcinoma found on histological examination of excised cysts $[3,6,8,9]$. However, the reported incidence of malignant transformation is only $0.045 \%$ [6]. The mechanism for malignant transformation has not been elucidated given its rarity; however, chronic irritation and repetitive trauma have been suggested to play a role [1].

We present the case of a large epidermoid cysts occurring over the olecranon process of the elbow, which seems to be previously unreported.

\section{Case Report}

A 31 year old male presented with a large, painless mass just lateral to his left olecranon process. The mass had been present for several years and had been steadily increasing in size, with little symptomatology. Notably, the patient had previously had a similar mass removed from his head/neck region, where a similar minimally symptomatic lump had slowly developed over the years. There was no history of trauma or previous surgery to the neck or elbow, normal motion, and no abnormal findings on plain radiography. Both neck and elbow swellings were surgically removed due to the patient's concern and their 
slow increase in size over several years. Clinical examination of the elbow lump revealed a cold mass, with deep tethering, but mobile superficial skin (Figure 1). A surgical incision was made directly over the mass and an approximately $5 \mathrm{~cm} \times 4 \mathrm{~cm}$ cystic structure was excised en bloc, and the tough fibrous adherence to the subcutaneous border of the ulna was completely removed by periosteal excision (Figures 2 and 3). This structure was incised on the back table and a large amount of greenish caseous material was found (Figure 4). The entire specimen was sent to for histological analysis. Upon examination, the pathologist found the mass to be a cystic structure lined by bland squamous epithelium with abundant keratinous debris. Furthermore, there was also a foreign-body giant cell reaction and focal cholesterol clefts, which are consistent with prior rupture. Notably, there was no evidence of malignancy noted (Figure 5).

\section{Discussion}

This case represents an unusual presentation of an epidermoid cyst to the orthopaedic clinic. Its location, in near proximity to the olecranon bursa could cause confusion as bursitis, a common entity in orthopedic clinics. It is important to recognize that the cyst did not involve the bursa and these are two distinct pathologies, which are managed very differently. Olecranon bursitis is the inflammation of the bursal sac which contains synovial fluid and provides virtually frictionless motion between the olecranon and the thin overlying soft tissue [10]. Generally, it presents as edema directly over the olecranon with minimal tenderness. There may or may not be erythema and warmth depending on the sterility of the bursitis and normal range of motion is preserved [11, 12]. Initial management of olecranon bursitis requires aspiration, application of compressive dressings, and non-steroidal antiinflammatory agents [13]. This treatment algorithm would be inadequate for an epidermoid cysts and aspiration may result in false assumption of septic olecranon bursitis as the caseous keratin debris can appear similar to purulence. Laboratory tests would correct the diagnosis but the patient may be started on unnecessary antibiotics prior to this correction. Additionally, as with all true cysts, complete excision of the cyst wall is needed to ensure that it does not recur and this is best done by removing an intact, circumferentially defined, mass. Cysts about the elbow

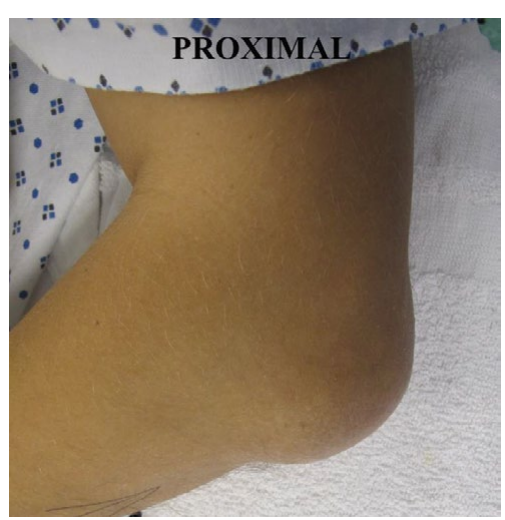

Figure 1 Preoperative photograph showing the mass over the olecranon.
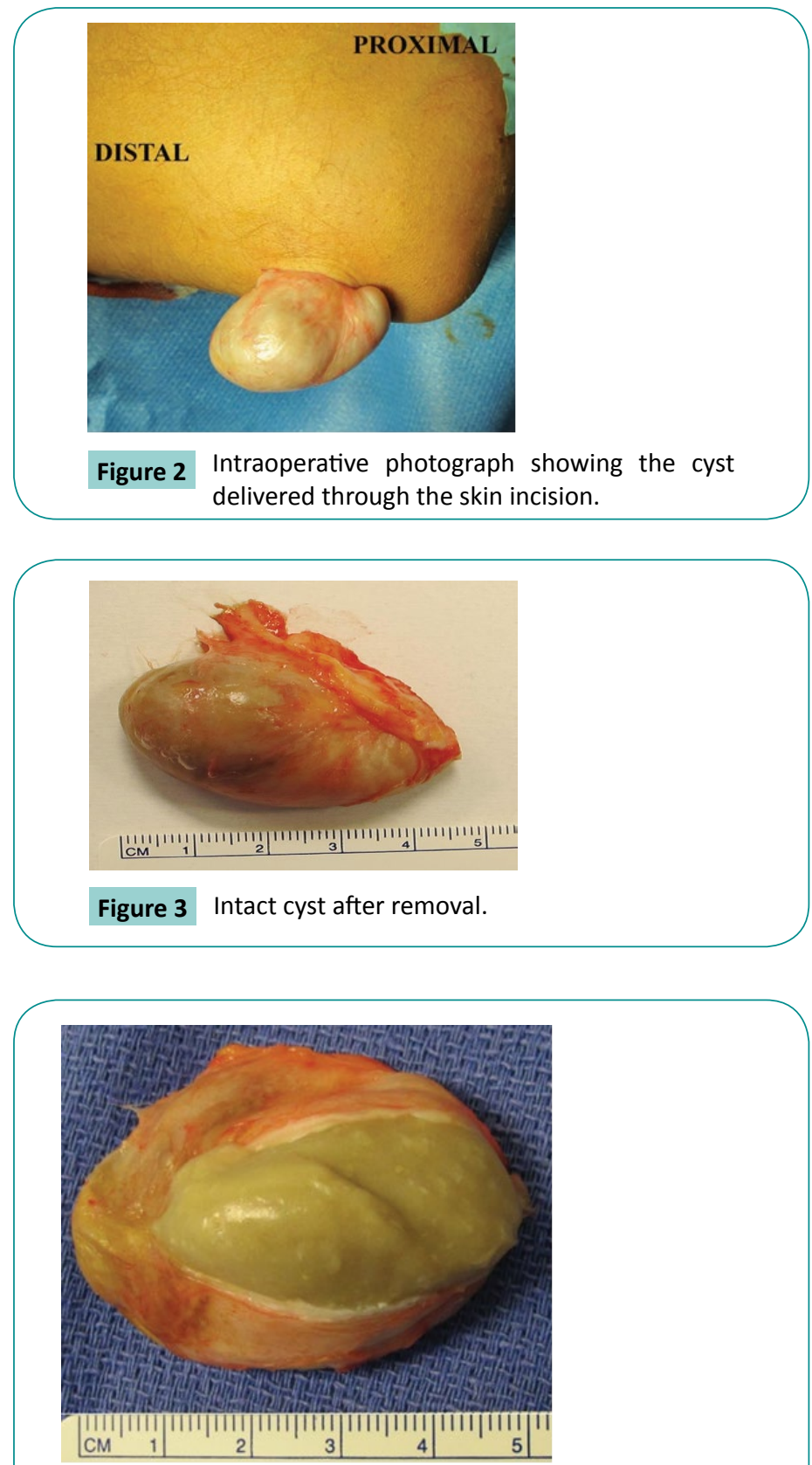

Figure 4 Cyst after it was opened on the back table of the OR, demonstrating the green, cheese-like substance that was contained inside.

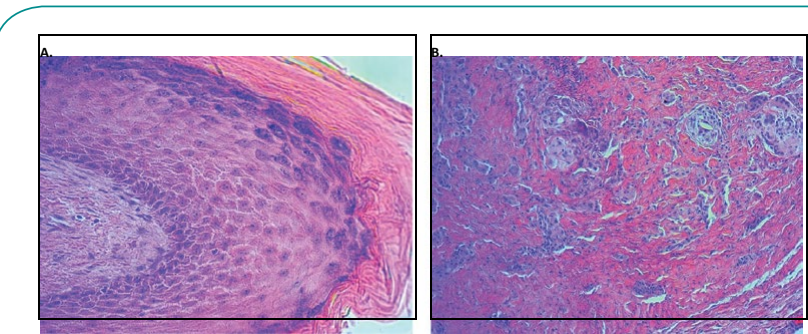

Figure 5 Histological sections of the removed cyst. A. Section demonstrating the bland squamous epithelium lining the cyst. B. Section demonstrating keratin debris with foreign body giant cell reaction. There was no histological evidence of malignancy. 
are uncommon and usually they are ganglia or synovial cysts, but it is important to keep this possibility in mind, as they do occur as seen in our case $[14,15]$. It is also important to remember that, although rare, malignant transformation of benign cysts does occur and therefore, all excised cysts should be sent for routine histological examination.
We present a rare presentation of a subcutaneous epidermoid cyst in close proximity to the olecranon bursa. It is important to recognize that this mass was separate from the bursa on physical examination so that en block excision could be performed. Additionally, all excised cysts should be sent for routine histological evaluation as malignant transformation is possible, although rare. 


\section{References}

1 Fromm LJ, N Zeitouni (2015) Epidermal Inclusion Cyst.

2 Henderson MM, MW Neumeister, RA Bueno (2014) Jr., Hand tumors: I. skin and soft-tissue tumors of the hand. Plast Reconstr Surg 133 154e-164e.

3 Ziadi S, et al. (2010) Squamous cell carcinoma arising from an epidermal inclusion cyst: A case report. N Am J Med Sci 2: 46-47.

4 Bhatt V, M Evans, TJ Malins (2008) Squamous cell carcinoma arising in the lining of an epidermoid cyst within the sublingual gland--a case report. Br J Oral Maxillofac Surg 46: 683-685.

5 Hayes D (1982) Traumatic epidermoid cyst of the ulna.

6 Cameron DS, RL Hilsinger Jr (2003) Squamous cell carcinoma in an epidermal inclusion cyst: case report. Otolaryngol Head Neck Surg 129: 141-143.

7 Patel K, et al. (2006) Epidermal inclusion cyst of phalanx: a case report and review of the literature. Skeletal Radiol 35: 861-863.
8 Chiu MY, ST Ho (2007) Squamous cell carcinoma arising from an epidermal cyst. Hong Kong Med J 13: 482-484.

9 Lopez-Rios F, et al. (1999) Squamous cell carcinoma arising in a cutaneous epidermal cyst: case report and literature review. Am J Dermatopathol 21: 174-177.

10 Wasserman AR, LD Melville, RH Birkhahn (2009) Septic bursitis: a case report and primer for the emergency clinician. J Emerg Med 37: $269-272$.

11 Blackwell JR, et al. (2014) Olecranon bursitis: a systematic overview. Shoulder \& Elbow 6: 182-190.

12 Reilly JP, JA Nicholas (1987) The chronically inflamed bursa. Clin Sports Med 6: 345-370.

13 McAfee JH, DL Smith (1988) Olecranon and prepatellar bursitis. Diagnosis and treatment. West J Med 149: 607-610.

14 Singh RA, et al. (2014) Unusual cases of elbow locking due to synovial cysts: a report of two cases. Bull Hosp Jt Dis (2013) 72: 308-310.

15 Zarezadeh A, et al. (2012) Intraosseous ganglion cyst of olecranon. Int J Prev Med 3: 581-584. 